\title{
The rise of epigenetic drug discovery
}

First draft submitted: 3 August 2016; Accepted for publication: 4 August 2016; Published online: 7 September 2016

Keywords: acetylation $\bullet$ epigenetics $\bullet$ histones $\bullet$ methylation

\section{What is 'epigenetics'?}

Epigenetics refers to changes in the genome that affect gene function without altering the nucleotide sequence of DNA. This special issue focuses on medicinal chemistry applied to epigenetic targets, one of the fastest growing areas of drug discovery in recent years. Epigenetic enzymes are responsible for covalent, but reversible, modification of chromatin in processes known as 'chromatin remodeling. Frequent targets for epigenetics enzymes are histone proteins, which are packaged with DNA to form chromatin [1]. DNA itself can also be directly modified by epigenetic enzymes, for example, cytosine methylation, and DNA methyltransferase inhibitors such as 5-aza-cytidine have been developed [2]. Additionally, miRNAs are increasingly implicated in gene regulation and are useful biomarkers. Covalent modifications carried out by epigenetic enzymes include methylation, acetylation, phosphorylation, ubiquitination and sumoylation. Epigenetic enzymes can be classified as 'readers' (e.g., bromodomains - protein domains in epigenetic enzymes which recognize acetlyated lysines), 'writers' (e.g., methylating or acetylating enzymes) and 'erasers' (e.g., demethylases or deacetylases). Depending on the disease, any of these processes, or a combination, could be aberrant in a cell leading to a wealth of druggable targets for researchers to exploit [3].

\section{Epigenetic targets have yielded drugs with more to come}

Epigenetic processes are implicated in a wide range of diseases especially cancer where tumor suppressor genes may be silenced and oncogenes may be activated [4]. Histone deacetlyases (HDACs) are the most advanced target class in terms of drug discovery with several compounds on the market, SAHA (vorinostat) being the first to be approved by the US FDA in 2006 [5]. However, drug developers have struggled for a long time to find wide applicability for HDAC inhibitors in cancer and the general trend is now to use them in combination with other drugs, for example, FDA-approved HDAC inhibitor panobinostat, in combination, for the treatment of multiple myeloma. In this issue, a promising class of phenylpyrrolebased HDAC inhibitors is reported, with HDAC1/6 activity in cells. Selective HDAC inhibition is a growing area where specific inhibition of one or more HDAC isozymes may improve the therapeutic window and open up new indications, such as neurodegenerative diseases. From this point of view, this issue includes an update on HDAC8 selective inhibitors.

More recently, inhibitors of bromodomains [6] and methylating enzymes have received considerable attention from all corners of the therapeutic community (see the 'bromone' editorial (Müller S) in this issue). A number of such compounds are in clinical trials, for example, birabresib (OTX015), a selective inhibitor of BET bromodomains is being studied in patients with both solid tumors and hematological malignancies. In this issue, bromodomain chemical probes as well as structural aspects of readers of methyl lysines are reviewed. Furthermore, this issue

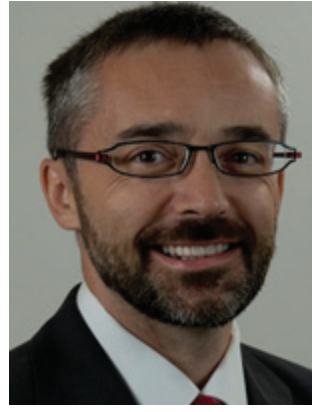

Brian W Dymock Department of Pharmacy, University of Singapore, Faculty of Science, Singapore 117543, Singapore

phadbw@nus.edu.sg 
describes new research toward JmjC domain-containing histone demethylases, a rapidly growing subfield of epigenetic 'erasers'.

Previous reports have highlighted progress toward histone methyltransferase inhibitors [7] and an update on the considerable progress being made in this area is reported in this issue, where the first histone methyltransferase inhibitor of EZH2 to enter the clinic, tazemetostat (EPZ-6438), for the treatment of $\mathrm{B}$-cell lymphomas, is discussed along with a progress update on the 'hot' fields of EZH2 and G9a inhibition. Another exciting new methyltransferase target, $\mathrm{H} 3 \mathrm{~K} 36$, and the challenges for inhibitor design are also reviewed.

Histone kinases, which phosphorylate specific histone residues, are another example of an exciting new target for anticancer drug discovery. In this issue, new research describing PRK1 inhibitors for prostate cancer based on the marketed JAK kinase inhibitor tofacitinib is reported.

\section{References}

1 Ryan L, Stesco N, Amhad TK, Cortes C, Namaka MP, Lakowski TM. Inhibitors of enzymes catalyzing modifications to histone lysine residues: structure, function and activity. Future Med. Chem. 8(8), 879-897 (2016).

2 Guianvarc'h D, Arimondo PB. Challenges in developing novel DNA methyltransferases inhibitors for cancer therapy. Future Med. Chem. 6, 1237 (2014).

3 Arrowsmith CH, Bountra C, Fish PV, Lee K, Schapira M. Epigenetic protein families: a new frontier for drug discovery. Nat. Rev. Drug Disc. 11, 384 (2012).

\section{Conclusion}

Epigenetics is now a major area for small molecule drug discovery with new epigenetic targets being validated with selective inhibitors reported on a regular basis. Now that more compounds are entering clinical testing, the power of epigenetic modification is one step closer to benefiting patients. This issue of Future Medicinal Chemistry presents a balanced coverage across this exciting field and will give the reader an excellent overview.

\section{Financial \& competing interests disclosure}

The authors have no relevant affiliations or financial involvement with any organization or entity with a financial interest in or financial conflict with the subject matter or materials discussed in the manuscript. This includes employment, consultancies, honoraria, stock ownership or options, expert testimony, grants or patents received or pending, or royalties.

No writing assistance was utilized in the production of this manuscript.

4 Lundstrom $\mathrm{K}$. What is the potential of epigenetics in drug development? Future Med. Chem. 7, 239 (2015).

5 Giannini G, Cabri W, Fattorusso C, Rodriquez M. Histone deacetylase inhibitors in the treatment of cancer: overview and perspectives. Future Med. Chem. 4, 1439 (2012).

6 Galdeano C, Ciulli A. Selectivity on-target of bromodomain chemical probes by structure-guided medicinal chemistry and chemical biology. Future Med. Chem. 8(13), 1655-1680 (2016).

7 Baugé C, Bazille C, Girard N, Lhuissier E, Boumediene $\mathrm{K}$. Histone methylases as novel drug targets: developing inhibitors of EZH2. Future Med. Chem. 6, 1943 (2014). 